aktivieren oder nicht beeinflussen, die Photosynthese hemmt. Nachdem L e h n in g e ${ }^{12}$ die Entkoppelung der Atmungskettenphosphorylierung durch DNP nachgewiesen hat, ist auch die DNP-Wirkung bei der Photosynthese zweifellos als eine Entkoppelung der Atmungskettenphosphorylierung zu deuten. Das Ausbleiben der Photosynthese bei DNP-Zusatz ist dann

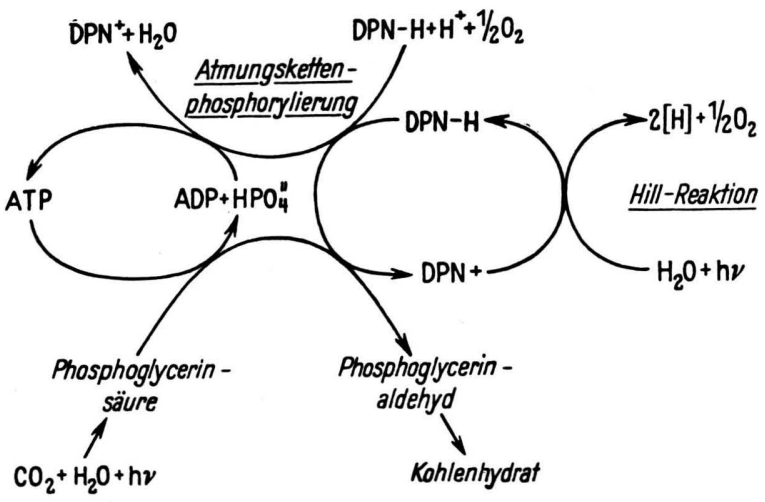

Abb. 3. Umkehrung der Triosephosphatdehydrierung bei der Photosynthese.

eine Folge der mangelnden Nachlieferung von ATP und Wegschaffung von anorganischem Phosphat und ADP.

Abb. 3 zeigt schematisch das Zusammenwirken 25 D. B u rk u. O. Warburg, Naturwiss. 37,560 [1950]. von DPN-Hydrierung (H ill-O choa-Reaktion) und Atmungskettenphosphorylierung bei der photosynthetischen Umkehrung der Triosephosphatdehydrierung.

Die speziell für die Triosephosphatdehydrierung diskutierten Verhältnisse sind sicher auch bei anderen Zwischenreaktionen der Photosynthese von Bedeutung. Die Triosephosphatdehydrierung wurde den Überlegungen zugrunde gelegt, da hier die Einzelheiten des Reaktionsverlaufes durch die Arbeiten von W a r bu r g bekannt sind.

Weiterhin gibt die diskutierte Beteiligung der Atmungskettenphosphorylierung bei der Photosynthese eine zwanglose Erklärung für den Befund von Burk und Warburg ${ }^{25}$, wonach bei der Photosynthese 3 Lichtquanten 3 Moleküle $\mathrm{CO}_{2}$ hydrierend fixieren und $2 / 3$ der in diesem primären Produkt festgelegten Energie durch Verbrennung wieder zur Verfügung gestellt werden, um in einem Kreisprozeß das dritte Molekül $\mathrm{CO}_{2}$ auf die Stufe der Glucose zu hydrieren. Nimmt man nämlich an, daß die beim Verbrennungsvorgang freigesetzte Energie durch den Mechanismus der Atmungskettenphosphorylierung zur Bildung von ATP benützt wird und in dieser Form als Energiedonator für die Überführung des dritten Moleküls $\mathrm{CO}_{2}$ auf die Stufe des Kohlenhydrates dient, so wird verständlich, daß das die Atmungskettenphosphorylierung entkoppelnde DNP einerseits die Dunkelatmung nicht beeinflußt bzw. aktiviert, und andererseits die Photosynthese hemmt.

\title{
Zur Gewebedifferenzierung des Grindelia-Blattes, insbesondere seiner Epidermis
}

\author{
Von Klaus Napp-ZinN \\ Aus dem Botanischen Institut der Universität Mainz \\ (Z. Naturforschg. 6 b, 430-437 [1951]; eingegangen am 23. August 1951)
}

Die Blattepidermis von Grindelia squarrosa führt Spaltöffnungen nur in den Bezirken über dem ober- und unterseits in kleinen Gruppen angeordneten Palisadenparenchym. Diese Gruppen werden durch einschichtige chlorophyllärmere Zellbänder getrennt, welche die Leitbündel mit der ober- und unterseitigen Epidermis verbinden, die dort niemals Stomata, sondern nur Emergenzen (Haare, ungestielte oder - seltener - gestielte Drüsen) aufweist.

Die Entwicklungsgeschichte zeigt, daß dies Muster auf einem frühen Stadium durch die Entstehung von Leitbündel- (und Sekretkanal-) Mutterzellen endgültig festgelegt wird, die die ersten Mesophyll-Zellteilungen außerhalb des Randmeristems in ihrer Richtung und gleichzeitig auch die gesamte weitere Gewebedifferenzierung bestimmen. Das Spaltöffnungsmuster stellt daher ein negatives Abbild des Leitbündelsystems dar. Ein Kippvorgang, der nach Art des von B ü n n ing und S a g r o m s k y bei Karatas Carolina erschlossenen erst auf einem relativ späten Stadium darüber entscheidet, ob aus einer bestimmten Initiale ein Haar oder eine Spaltöffnung hervorgeht, kommt bei Grindelia nicht in Betracht. 
Die durch Wuchsstoffpaste (K-Salz der $\beta$-Indolylessigsäure in 10-3-mol. Lösung) erzielten Abweichungen in der Ausbildung der Epidermis harmonieren mit den Ergebnissen von B ü n $\mathrm{n}$ ing und Sagromsky bei Impatiens Sultani. Im einzelnen handelt es sich dabei um Unterdrückung der Spaltöffnungsbildung, Schädigung bereits vorhandener Stomata, Auslösung eines Zellteilungsschocks und anschließende Steigerung der Spaltöffnungsinitialenbildung.

Abschließend werden die stofflichen Voraussetzungen der Stomata- und Emergenzenbildung im Hinblick auf Wuchsstoffeinfluß und Aciditätsverhältnisse diskutiert.

S eitdem Bünning und Sa gromsky ${ }^{1,2}$ Hinweise auf die stoffliche Beeinflussung der Stomatabildung geben konnten, hat die Frage nach der Entstehung der Spaltöffnungsmuster in der Blattepidermis neuen Auftrieb erhalten; es erscheint daher wünschenswert, Blätter mit charakteristischen Epidermismustern daraufhin zu studieren, ob sie weitere Anhaltspunkte zu den von den genannten Autoren entwickelten Vorstellungen liefern.

\section{Anatomische Verhältnisse}

Bei früheren Untersuchungen unter anderen Gesichtspunkten $^{3}$ fiel ein Objekt auf, das zu einer Erforschung im angedeuteten Sinne besonders geeignet erschien, nämlich die zur Solidago-Verwandtschaft gehörige Composite Grindelia squarrosa Dus. Zur Darstellung der an dieser Pflanze gewonnenen Ergebnisse gehen wir aus von den Verhältnissen, wie wir sie beispielsweise an einem vollentwickelten Lauboder Hochblatt antreffen. Zieht man einem solchen Blattorgan die ober- oder unterseitige Epidermis ab, so stellt man zunächst fest, daß die Spaltöffnungen in deutlichen Gruppen zusammenliegen, die durch Streifen stomatafreier Zellen getrennt sind (Abb. 1 a). Anhängende Reste des darunter befindlichen Mesophylls weisen darauf hin, daß die erwähnten Gruppen von Spaltöffnungen sich nur in denjenigen Teilen der Epidermis finden, unter denen das Mesophyll als Palisadenparenchym ausgebildet ist. Dickere Flächenschnitte durch Grindelia-Blätter lassen die meist in Zwei- oder Mehrzahl unter den spaltöffnungsfreien Epidermisstreifen liegenden Gefäße erkennen, die sich durch die Phloroglucin/HCl-Reaktion unschwer hervorheben lassen (Abb. 1b)*.

Mehrzellige Haare, kleine gestielte und vielzellige ungestielte Drüsen (Abb. 2) finden sich nur in den zu-

1 E. Bünning u. H. S a gromsky, Z. Naturforschg. 3 b, 203 [1948].

2 H. S a g r o m s k y, Z. Naturforschg. 4 b, 360 [1949].

3 K. N a p p - Z i n n, Österr. bot. Z. 98, 142 [1951].

* Der entgegengesetzte Fall (Spaltöffnungen vorzugsweise über Leitbündeln) liegt nach $W$ e be ${ }^{4}$ bei der Sproßachse von Chirita Horsfeldii vor und scheint - nach unseren bisherigen Kenntnissen - eine seltene Ausnahme darzustellen. letzt erwähnten stomatalosen Teilen des Hautgewebes, was zunächst in Übereinstimmung mit den Befunden von Bünning und Sagromsky ${ }^{1}$ zugunsten der Annahme spricht, daß die über den Leit-
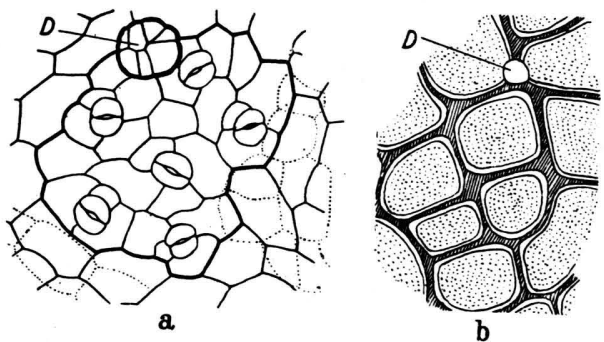

Abb. 1. Laubblattausschnitte von Grindelia squarrosa in Flächenansicht. a Epidermis der Oberseite; Rand des Spaltöffnungsfeldes durch stärkere Linien hervorgehoben; unter den stomatafreien Streifen liegende Zellen der „Aufhängebänder“ durch punktierte Umrißlinien angedeutet; b dickerer Schnitt bei schwacher Vergrößerung, schematisch; Leitbündel schraffiert, Spaltöffnungsfelder (über Chlorenchym) punktiert. $\mathrm{D}=$ Drüse.

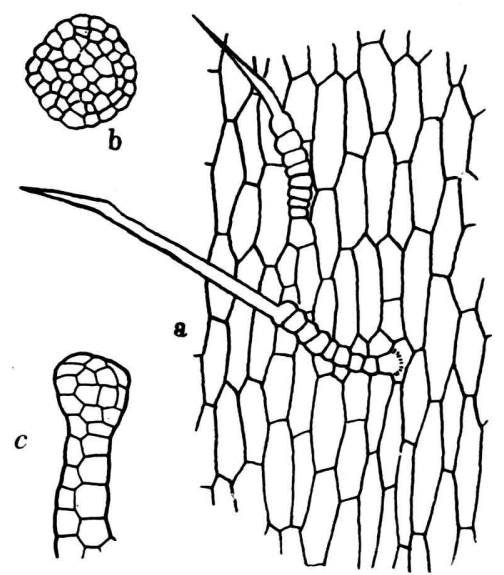

Abb. 2. Emergenzen des Grindelia-Laubblattes. a Haare der Mittelrippe; b vielzellige ungestielte Drüse in Oberansicht; c gestielte Drüse.

bündeln befindlichen Epidermispartien zumindest in der letzten Phase ihrer Differenzierung anderen (mit den Leitbündeln in Zusammenhang stehenden) stofflichen Einflüssen ausgesetzt sind als die übrigen Bezirke der Epidermis. 
Im einzelnen variiert die Natur der Blattemergenzen in den verschiedenen Sproßabschnitten manchmal nicht unerheblich. Bei den Involucralblättern dominieren ganz entschieden die relativ großen, ungestielten Drüsen, bei den Hochblättern sind diese mit zahlreichen kleineren, gestielten Drüsen durchmischt, und bei den Laubblättern schließlich herrschen letztere manchmal bei weitem vor; nur vereinzelt sind hier dann noch kugelige, ungestielte Drüsen anzutreffen, während sich Haare grundsätzlich nur im Medianbereich der Laubblätter, und zwar vorzugsweise auf dem Mittelnerven, vorfinden. - Es begegneten mir jedoch auch zahlreiche Exemplare von Grindelia squarrosa, deren Laubblätter nur ungestielte Drüsen trugen; überhaupt scheint bei dieser Pflanze eine große nung, wenn man es zusammen mit einem Blatt einer anderen Pflanze, etwa Chrysanthemum segetum, gegen das Licht hält und das „Geäder“ vergleicht.

Ergänzungsweise sei hier auch kurz auf die Sproßepidermis eingegangen. Das Hautgewebe dünnerer Stengel (etwa $1 \mathrm{~mm}$ Durchmesser), das größtenteils aus langgestreckten Zellen besteht, weist nur ganz vereinzelt Spaltöffnungen auf; das darunterliegende Rindengewebe ist allgemein kollenchymatisch ausgebildet. Haare von der Art der in Abb. 2 a wiedergegebenen finden sich hier häufiger.

Anders bei kräftigeren, etwa $3 \mathrm{~mm}$ dicken Sproßachsen: Hier treffen wir in der Epidermis zahlreiche streifenartige

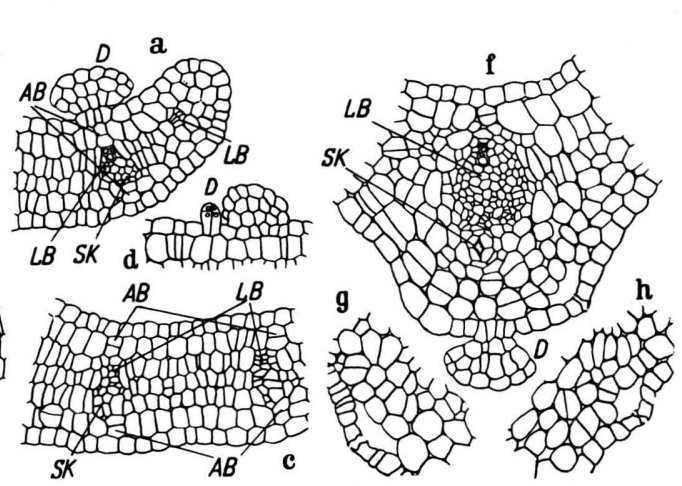

Abb. 4.

Abb. 3.

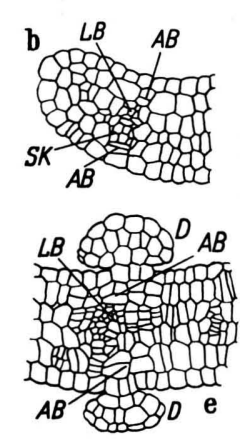

Abb. 3. Partieller Querschnitt durch ein (äquifaciales) Laubblatt; Leitbündel schraffiert, Chlorenchym punktiert. Abb. 4. Querschnitte durch junge Blätter. a-e veranschaulichen die Anlegung von Leitbündeln (LB), Sekretkanälen (SK) und Aufhängebändern (AB), $\mathrm{f}-\mathrm{h}$ zeigen die ersten Stomata. In c (in der Nähe des Medianus) bereits fünf Mesophyllschichten; in e Leitbündel- und Aufhängeband-Anlage zum Teil in Seitenansicht. Näheres im Text.

Variabilität zu herrschen, die sich z. B. auch in unterschiedlicher Wuchshöhe, Blattgröße, -gestaltung und -orientierung und Blütezeit manifestiert.

Blattquerschnitte (Abb. 3) veranschaulichen schließlich die „Aufhängung“ aller Leitbündel an den Epidermen mittels ober- und unterseitiger, chlorophyllarmer Mesophyllbänder, die das Palisadenparenchym in kleine Gruppen gliedern und den Vergleich mit Mesenterien nahelegen. - Auch bei den Blättern anderer Pflanzen pflegt freilich das Mesophyll in der Umgebung der Leitbündel, zumal im Bereich der Hauptnerven, chlorophyllärmer zu sein, doch ist mir bisher kein ähnliches Beispiel für derartige „Aufhängebänder" bekannt geworden, wie sie gerade bei Grindelia anzutreffen sind; allenfalls könnte man die Sklerenchymstränge, die z. B. bei Gramineenblättern zwischen Leitbündeln und Epidermis liegen, in Betracht ziehen. - Diese Besonderheit des GrindeliaBlattes tritt übrigens schon makroskopisch in Erschei-

4 H. W e b e r, S.-B. Heidelb. Akad. Wiss., Math.naturwiss. Kl. 1949, 161.
Gruppen von zwei oder drei Spaltöffnungen an, wie sie ähnlich von W e be ${ }^{4}$ für Silphium perfoliatum und Aster beschrieben wurden. Unter diesen Stomatafeldern, die übrigens niemals Haare enthalten und im Gegensatz zu den übrigen Epidermiszellen stets anthocyanfrei bleiben, liegt ein charakteristisches Schwammgewebe; dabei ist hier die Rinde, die auch Ansätze zur Lentizellenbildung zeigt, kaum dicker als bei schwächeren Sprossen. Die in den spaltöffnungsfreien Epidermisbezirken stärkerer Sprosse inserierten Haare weichen insofern von denjenigen der Blattrippen und dünneren Stengel ab, als sie erheblich länger und im wesentlichen aus zur Spitze hin allmählich größer werdenden Zellen zusammengesetzt sind, die die Basalzellen der Haare von Blattnerven und schwachen Sprossen um etwa das Doppelte an Länge übertreffen.

\section{Entwicklungs ges chichte}

Über den zeitlichen Ablauf der im vorhergehenden geschilderten charakteristischen Differenzierung von Mesophyll und Epidermis des Grindelia-Blattes gibt allein die Entwicklungsgeschichte Auskunft. Wie bei Dicotylenblättern üblich, herrscht auch hier ein ausgesprochenes Randwachstum, das ein zunächst vier- 
schichtiges Mesophyll entstehen läßt (Abb.4a). Auf sehr frühen Stadien und in geringer Entfernung vom Blattrand erfolgt bereits die Anlegung der Leitbündel durch Aufteilung jeweils einer im Querschnitt sichtbaren Zelle der zweitobersten Mesophyllschicht, während die Anlage von Sekretkanälen, welche manche Leitbündel begleiten, in anfangs ganz ähnlicher Weise durch Zerlegung je einer Zelle der zweituntersten Mesophyll-Lage vonstatten geht (Abb.4b). (Selbstverständlich handelt es sich jeweils um kontinuierliche Züge von Leitbündel- und Sekretkanalmutterzellen, von denen aber auf Querschnitten naturgemäß nur je eine in Erscheinung treten kann.)

Mit der Entstehung der Leitbündelanlagen, dem offenbar ersten Differenzierungsprozeß im Blattprimordium, scheint die gesamte Weiterentwicklung des Blattes bereits festgelegt zu sein, erweisen sich doch die nächstfolgenden Zellteilungen - die ersten, die außerhalb des Randmeristems in der Umgebung der Bündelanlagen im Mesophyll stattfinden - als durchweg von der nächstliegenden Leitbündelmutterzelle in ihrer Richtung determiniert: Die über der Leitbündelanlage befindliche Mesophyllzelle teilt sich ebenso wie die unter der Bündel- bzw. Sekretkanalinitiale gelegene Zelle der untersten Mesophyllschicht stets zuerst periklinal (in bezug auf die Blattoberfläche) und hält im allgemeinen an dem periklinalen Teilungsmodus bis zum Abschluß der Blattentwicklung fest. Auf diese Weise entstehen die oben erwähnten, im Querschnitt einzellreihigen „Aufhängebänder“. Nur in Ausnahmefällen wird in deren Entwicklung eine (im Querschnitt erkennbare) antiklinale Teilung eingeschoben, was dann die stellenweise zweireihige Ausbildung eines Bandes zur Folge hat.

Die seitlich an die Sekretkanal- und Leitbündelinitialen angrenzenden Mesophyllzellen hingegen teilen sich zunächst im allgemeinen antiklinal ${ }^{1}$ und gehen erst dann zu periklinalen Teilungen* über, welche späterhin mit antiklinalen abwechseln. - Die übrigen, nicht über oder unter Leitbündel- und Sekretkanalanlagen befindlichen Zellen der obersten und untersten Mesophyllschicht pflegen schließlich in ihrer Mehrzahl in der Nähe der künftigen „Aufhängebänder" zuerst periklinale, sonst aber meist antiklinale Teilungen zu erfahren.

Auf den hier geschilderten frühen Entwicklungs-

* Stets auf die Blattoberfläche bezogen.

** Im übrigen liegen in Blattgrund und Spreite der Hüllblätter von Grindelia in mancher Hinsicht abweichende Verhältnisse vor, auf die wir hier nicht näher eingehen können. stadien, in denen bereits die grundlegende Mesophylldifferenzierung erfolgt, ist die Epidermis noch vergleichsweise arm an charakteristischen Strukturen. Als erstes erfolgt - etwa gleichzeitig mit den ersten Teilungen der Leitbündelinitialen - die Anlage von Drüsen (in der Epidermis über und unter den Bündelmutterzellen), deren Initialen sich rasch in vier Quadranten teilen, von denen (bei großen, ungestielten Drüsen) die beiden basalen diejenigen Zellen liefern, die das aus den oberen Quadranten entstehende Köpfchen in der Epidermis verankern. Die Entwicklung der Drüsen vollzieht sich so schnell, daß diese sich bei einer etwa 3-4 mm langen Blattanlage gegenseitig oft berühren; während bei den Laubblättern

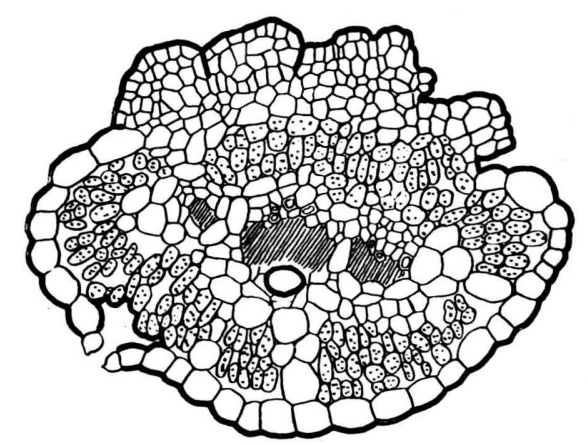

Abb. 5. Querschnitt durch die Spitze eines äußeren (laminalen) Involucralblattes.

die Abstände zwischen ihnen im allgemeinen durch späteres Wachstum erheblich vergrößert werden, bleiben sie z. B. bei den laminalen Abschnitten der Involucralblätter fortwährend in Kontakt (Abb. 5) **. Überhaupt scheint von den Drüsen keine solche Hemmwirkung auszugehen, wie sie B ünning und $\mathrm{Sagromsk}{ }^{1}$ und Weber ${ }^{4}$ bei Spaltöffnungen bzw. Stomatagruppen fanden; dies ist auch aus der Tatsache zu schließen, daß gelegentlich in unmittelbarer Nähe einer Drüse nachträglich eine zweite entsteht (Abb. 4d).

Wie schon B ünning und S a grom sky ${ }^{1 a}$ feststellten, treten die Zellteilungen, aus denen die Spaltöffnungsinitialen hervorgehen, erst dann ein, wenn die übrige Teilungstätigkeit in der Epidermis ganz oder vorübergehend erloschen ist. So auch bei Grindelia, an deren Blättern die ersten Stomata freilich nur in Querschnitten zu erkennen sind, da die zahlreichen Drüsen den Blick auf die Epidermis versperren. Auf einem Schnitt durch ein etwa $1,1 \mathrm{~mm}$ breites (in Knospenlage eingerolltes) Blatt erkennt man deut- 
lich, daß die ersten Spaltöffnungen auf der Unterseite in der Nähe des Medianus gebildet werden und mit der Interzellularenbildung in Zusammenhang stehen (Abb. $4 \mathrm{f}-\mathrm{h}$ ), die ihrerseits wiederum nur die Mutterzellen des Palisadengewebes, niemals aber die „Aufhängebänder“ betrifft. — Das bei der Stomatabildung nur geringfügig gesteigerte Epidermis-Wachstum scheint jedoch zur Erklärung der lokalen Abhebung der Epidermis vom Mesophyll und einer dadurch bedingten Entstehung von Zwischenzellräumen bei diesem Objekt nicht auszureichen. Vielmehr spricht manches dafür, daß im Bereich einer in Entwicklung begriffenen Spaltöffnung das Mesophyllwachstum gehemmt ist (vgl. Abb. 4f, h). - Bei etwas älteren Blät-

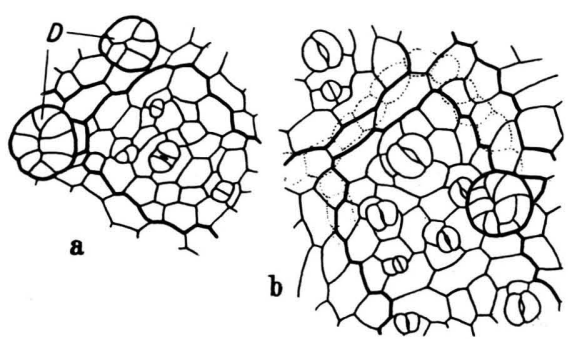

Abb. 6. Epidermis-Ausschnitte aus 6,5 mm langem Laubblatt (Oberseite). a etwa aus der Mitte, b mehr distal. Darstellung wie in Abb. 1 a.

tern, die bereits eine Flächenansicht gestatten, findet man häufig in einer Spaltöffnungsgruppe ein oder zwei größere Stomata neben mehreren offenkundig erst später entstandenen kleineren (Abb. 6; vgl. Alliaria officinalis nach Bünning u. Sagromsky ${ }^{1}$ ).

Auf Grund der bisher geschilderten Befunde läßt sich nunmehr die von den genannten Autoren aufgeworfene Frage nach der Abbild-Natur des Spaltöffnungsmusters dahingehend beantworten, daß dieses - wenigstens bei Grindelia - unbedingt ein Abbild - und zwar gewissermaßen ein negatives Abbild — des Leitbündelnetzes darstellt. Wodurch dessen Entstehung wiederum induziert wird, scheint vorläufig noch ungeklärt. Bei Bünnings neuerlichen Untersuchungen über die Differenzierungsvorgänge in der Cruciferenwurzel ${ }^{5}$ fällt gerade den Hadromprimanen eine entscheidende Rolle für die weitere Histogenese zu.

Im übrigen sprechen unsere Befunde an Grindelia hinsichtlich der Reihenfolge der die Epidermisdifferenzierung bestimmenden Kippvorgänge für die Möglichkeit einer anderen Auffassung neben der von

5 E. B ü nning, Planta (Berl.) 39, 126 [1951].
B ünning und Sagromsky ${ }^{1}$ auf Grund ihrer Beobachtungen entwickelten; und zwar legt die frühzeitige Determinierung der Epidermiszonen durch die Leitbündel folgende Deutung nahe: Die Epidermiszellen über und unter Leitbündel-(und Sekretkanal-) Anlagen haben die Möglichkeit, sich entweder zu Haaren, ungestielten vielzelligen oder gestielten wenigzelligen Drüsen einerseits - oder aber zu „normalen“ Epidermiszellen andererseits zu entwickeln; die Zellen der über Chlorenchymanlagen befindlichen Epidermisbezirke dagegen können entweder Spaltöffnungen oder auch "normale“ Epidermiszellen liefern. Ein Kippvorgang, dem die auf je eine Initialzelle beschränkte Alternative: Spaltöffnung oder Emergenz, zugrunde liegt, wie ihn Bünning und $\mathrm{S}$ a g r o m s k ${ }^{1}$ vor allem auf Grund ihrer Befunde an Karatas Carolina an das Ende der Epidermisdifferenzierung stellen, kommt demnach bei Grindelia wohl kaum in Betracht.

\section{Versuche zur Beeinflussung der Epidermisdifferenzierung durch Wuchs toffpaste}

Im Anschluß an die vorstehend wiedergegebenen entwicklungsgeschichtlichen Studien wurde eine kleine Versuchsreihe durchgeführt, die lediglich orientierenden Charakter trug und nicht zur statistischen Auswertung vorgesehen war.

Mehrere in Freilandkultur befindliche, möglichst gleichartige Grindelia-Sprosse wurden etwa $30-35 \mathrm{~cm}$ über dem Boden dekapitiert; alsdann wurden an jedem dieser Sprosse die 8-10 obersten Achselknospen - soweit möglich auch zwischen den Blattanlagen - mit der gleichen Wuchsstoffpaste bestrichen, wobei $\beta$-indolylessigsaures Kalium an den verschiedenen Pflanzen entweder in 10-3-, 10-4-, 10-5- oder 10-6-mol. Lösung (neben einer Wasserkontrolle) zur Anwendung kam. Am 4., 7. und 1.1. Tage nach Versuchsbeginn wurde die Pastenauflage erneuert; die Ernte erfolgte am 11. und 20. Tag. Im allgemeinen wurden Epidermis-Ausschnitte aus der distalen Hälfte des mittleren Drittels von der Unterseite von je $10-151-2 \mathrm{~cm}$ langen Blättern untersucht.

Am deutlichsten war die Wirkung zweifelsohne bei 1.0 ${ }^{-3}$-mol. Heteroauxin-Lösung. Abb. $7 \mathrm{a}, \mathrm{b}$, die Ausschnitte aus einem halbseitig behandelten $22 \mathrm{~mm}$ langen Blatt darstellt, veranschaulicht fast alle Veränderungen, denen die Epidermis bei dieser Wuchsstoffkonzentration unterworfen sein kann: Zunächst ist in Úbereinstimmung mit den Befunden von B ü n $\mathrm{ning}$ und $\mathrm{Sagromsky}{ }^{1}$ und $\mathrm{Umrath}^{6}$ eine

6 K. U m r a th, Planta (Berl.) 36, 262 [1948]. 
weitgehende Unterdrückung der Spaltöffnungsbildung festzustellen, mit der oft eine auffällige Schädigung bereits vorhandener Stomata verbunden ist (vgl, auch Abb. $7 \mathrm{c}$ ). Die wenigen trotzdem entstandenen Spaltöftnungen sind im allgemeinen wesentlich kleiner als diejenigen der unbehandelten Epidermisbereiche. Darüber hinaus ist auch die Größe der übrigen Epidermiszellen - im Vergleich zu derjenigen nicht be-
Emergenzen nicht so sehr auf die Medianregion beschränkt wie sonst üblich.

Úber einen Einfluß der Wuchsstoffpaste auf die Bildung der kugeligen Drüsen (gestielte kamen bei dem Versuchsmaterial nicht vor) können keine Aussagen gemacht werden, da einerseits die Drüsen bereits entstanden sein werden, ehe das Blattprimordium mit Heteroauxin in Berührung kam, und
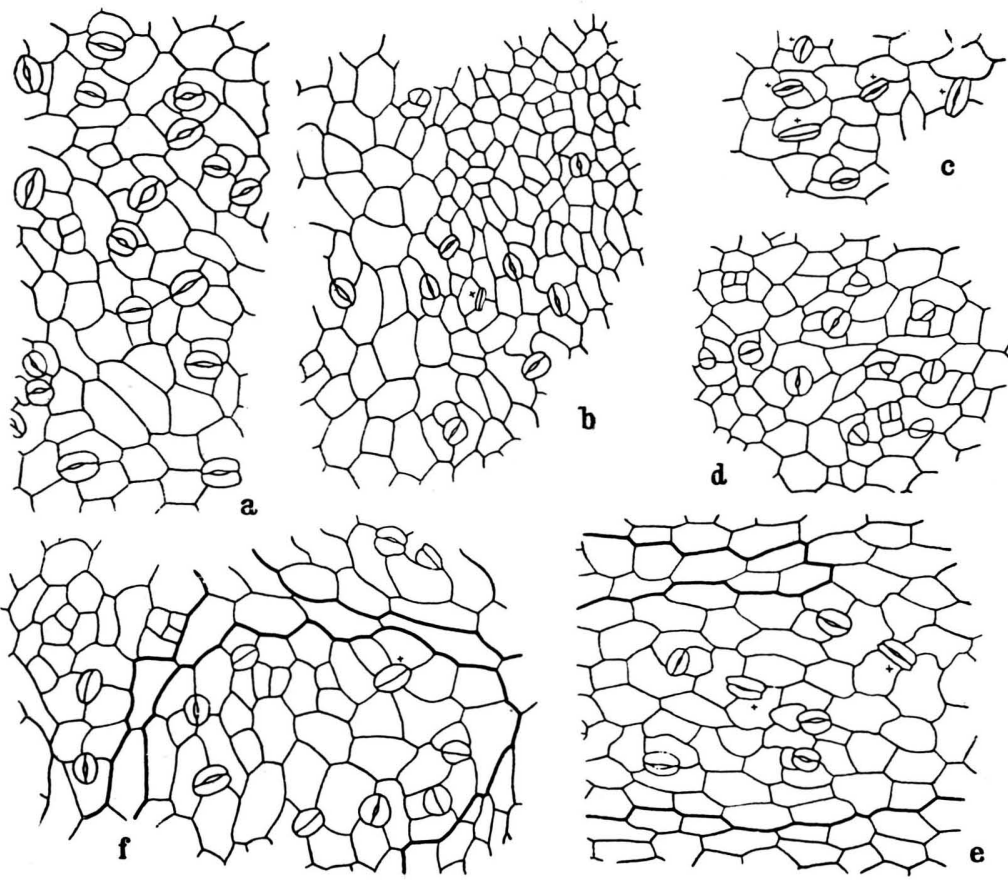

Abb. 7. Epidermis-Ausschnitte aus jungen Blättern, die mit $\beta$-indolylessigsaurem Kalium in 10-3-mol. Lösung behandelt worden waren. $\mathrm{a}, \mathrm{b}$ und e aus einem $22 \mathrm{~mm}$ langen Blatt (a unbehandelte, b und e behandelte Hälfte); c aus einem $24 \mathrm{~mm}$ langen Blatt (Schädigung bereits vorhandener Stomata); d aus einem 9 mm langen Blatt (Steigerung der Initialenbildung); f aus einem $12 \mathrm{~mm}$ langen Blatt. $+=$ abgestorbene und deformierte Spaltöffnung. Näheres im Text.

handelter Blattabschnitte - erheblich herabgesetzt, was auf vermehrte Teilungen in Verbindung mit verringertem Streckungswachstum zurückzuführen ist. (Die behandelte Hälfte des in Rede stehenden Blattes blieb schmäler als die unbehandelte, ohne aber deshalb auffällige Wachstumskrümmungen oder sonstige Mißbildungen zu zeigen, wie sie sonst bei der Konzentration $10^{-3}$ - und nur bei dieser - häufig auftraten.) Auch hier kam es früher oder später zur Steigerung der Spaltöffnungsinitialenbildung (Abb. $7 \mathrm{~d}$ ), sobald der durch die Wuchsstoffpaste induzierte Teilungsschock abgeklungen war. - Die Anzahl der Haare vom Typ der Abb. 2 a schien bei dieser Konzentration geringfügig gesteigert; auch blieben diese andererseits die Drüsendichte der Blattgröße etwa umgekehrt proportional ist*.

Schließlich muß noch erwähnt werden, daß die geschilderten Wuchsstoffwirkungen nicht immmer gleichzeitig auftraten, daß vielmehr meistens nur einige von ihnen kombiniert waren. Es sei darum an die

* Die Drüsenbildung wird hier nämlich offenbar endgültig abgeschlossen, ehe das Blatt vom Randwachstum zum (,intercostalen“) Flächenwachstum übergeht; beim Hochblatt wird letzteres mit steigender Insertionshöhe in zunehmendem Maße unterdrückt; so erklärt sich auch $\mathrm{N}$ e s s e s von $\mathrm{N}$ e t o lit z k y ${ }^{7}$ zitierte Beobachtung, daß bei den Hochblättern die Haare erheblich dichter stehen als auf den Laubblättern, und zwar um so mehr, je höher das Hochblatt inseriert ist.

7 F. Netolitzky, Die Pflanzenhaare. Berlin 1932. 
Versuche von $\mathrm{U} \mathrm{m} \mathrm{rat} \mathrm{h}^{6}$ erinnert, der bei der höchsten von ihm angewandten Konzentration $\left(10^{-4}-\mathrm{mol}\right.$.) gleichfalls unterschiedliche Wirkungen feststellte.

Bei Applikation von $10^{-4}$-mol. Heteroauxin-Lösung in Pastenform bot sich ein ganz anderes Bild dar: Größe und Anzahl der Stomata (Abb. $8 \mathrm{a}, \mathrm{c}$ ) waren nicht nur im Vergleich zur $10^{-3}$-mol. Lösung, sondern auch zur Kontrolle gesteigert (entsprechend dem von $\mathrm{U} \mathrm{m} r \mathrm{ath}$ bei Neptunia plena gefundenen Maximum bei der Konzentration $1 / 4,7 \cdot 10^{4}$ bzw. $\left.1 / 10^{5}\right)$. Geschädigte (deformierte) Spaltöffnungen waren niemals zu finden; eine auffällige Häufung von Stomata-Initialenbildungen oder ein zur Kleinheit der Zellen
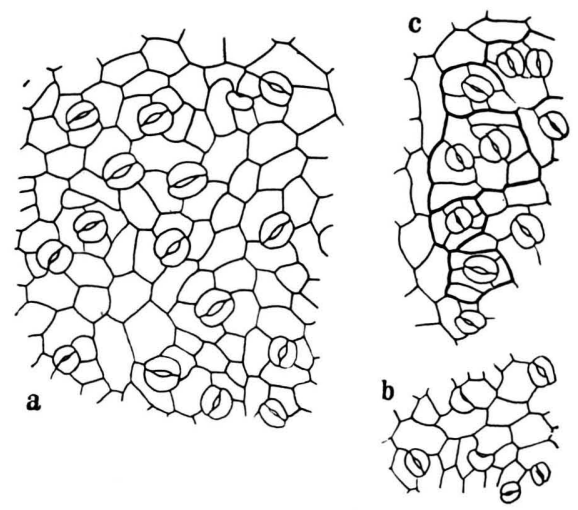

Abb. 8. Wie Abb. 7, jedoch 10-4-mol. Lösung. In a und b aus nur einer Schließzelle bestehende Stomata; in $\mathrm{c}$ sind einige Zellen stärker umrandet, durch deren Aufteilung Spaltöffnungen entstanden.

führender Teilungsschock waren weder nach 11 noch nach 20 Tagen zu beobachten; jedoch wurden ausschließlich bei dieser Verdünnungsstufe (insgesamt 5) Spaltöffnungen beobachtet, die aus nur einer Schließzelle bestanden (Abb. $8 \mathrm{a}, \mathrm{b})$.

Bei den beiden schwächsten Konzentrationen konnten ebensowenig wie bei der Wasserkontrolle wesentliche Unterschiede im Vergleich zu unbehandelten Blättern gefunden werden.

\section{Diskussion der Ergebnisse}

Unsere Kenntnisse über die stofflichen Voraussetzungen für die Stomatabildung lassen sich nun dahingehend zusammenfassen, daß diese einmal vom Wuchsstoffgehalt der Blattanlage, zum andern von den Aciditätsverhältnissen abhängig ist.

Hinsichtlich des Wuchsstoffeinflusses lassen sich unsere Befunde, wie bereits im vorigen Abschnitt angedeutet, recht gut mit denjenigen von Umrath, $\mathrm{B}$ ü $\mathrm{n} \mathrm{n} \mathrm{ing} \mathrm{und} \mathrm{S} \mathrm{a} \mathrm{g} \mathrm{r} \mathrm{o} \mathrm{m} \mathrm{s} \mathrm{ky} \mathrm{in} \mathrm{Einklang} \mathrm{bringen;}$ dabei dürfte auf weitere Sicht die von $\mathrm{U} \mathrm{m}$ r a th benutzte Methode (Aufzucht der Versuchspflanzen in der Heteroauxin-Lösung) gegenüber der Auftragung von Wuchsstoffpaste zu bevorzugen sein, da bei letzterer die tatsächliche Wuchsstoffaufnahme in das Blatt schwerer zu kontrollieren ist; bei ersterer stört freilich die kurzfristige Haltbarkeit der Versuchslösungen.

Im Hinblick auf die Aciditätsverhältnisse scheinen sich jedoch die Ergebnisse von U m rath einerseits und B ünning und S a gromsky andererseits zu widersprechen. Während bei letzteren die Erniedrigung der Acidität die Spaltöffnungsbildung deutlich förderte, wurde bei $\mathrm{Umrath}$ in einem ähnlichen Fall (bei Neutralisation der $\beta$-Indolylessigsäure durch Ammoniak) die Anzahl der Stomata vermindert. Zur Klärung dieser Frage werden daher weitere Untersuchungen erforderlich sein. Allerdings finden B ü n $\mathrm{n}$ ing s Befunde von vornherein eine gewisse Stütze durch die früheren Beobachtungen des gleichen Autors $^{8}$ über die Verschiedenheit des $p_{\mathrm{H}}$-Wertes auf Ober- und Unterseite des Phaseolus-Gelenks, doch müßte auch dies Resultat (oder besser: die Korrelation zwischen Basizität und Stomatazahl) durch Bearbeitung einer größeren Zahl von Arten auf Allgemeingültigkeit überprüft werden.

Ganz unabhängig von der zuletzt berührten Frage kann man schon jetzt sagen, daß die die Stomatabildung unterdrückenden stofflichen Agenzien an die Leitbündel (bzw. deren Mutterzellen) und ihre Umgebung gebunden sind. Zugunsten dieser Annahme läßt sich auch unsere Feststellung anführen, daß bereits durch die Bildung der Bündelinitialen das Netz der stomatafreien Epidermisstreifen prädeterminiert ist. Im übrigen spielt hier wahrscheinlich auch die Blattdicke (bzw. der Abstand zwischen Epidermis und Leitbündel) eine Rolle. Wie R. S chneider (unveröff.) fand, entstehen auch bei zahlreichen anderen Dicotylenblättern Leitbündel aus der zweitobersten von den vier Mesophyllschichten der jungen Blattanlage, also näher der später meist spaltöffnungsärmeren Oberseite. Zudem ist auch das nach $\mathrm{B}$ ünning ${ }^{5}$ eine Differenzierungs-Hemmzone bildende Hadrom der Oberseite zugewandt. Denkt man sich nun um jedes noch so kleine Leitbündel bzw. Hadrom (als Mittelpunkt) einen im Querschnitt kreisförmigen Hemmungsbezirk, so umfaßt dieser natürlich einen größeren Teil der ober- als der unterseitigen Epidermis.

Ferner spricht für unsere Vermutung die Tatsache,

8 E. B ünn ing, Z. Bot. 37, 433 [1942]. 
daß dickere Blattorgane zuweilen wesentlich mehr Spaltöffnungen pro Flächeneinheit besitzen als dünnere homologe. So weist z. B. der Blattgrund des Laubblattes von Villarsia parnassiaefolia zahlreiche Stomata auf, während der vaginale Hochblattabschnitt dieser Pflanze Spaltöffnungen gänzlich vermissen läßt.

Ist nun einmal durch die „mangelnde Einflußnahme" der durch ein Leitbündel bedingten Hemmungssphäre in einem bestimmten Epidermisbereich die Möglichkeit zur Stomatabildung freigegeben, so mag schließlich durch weitere Faktoren bestimmt werden, welche Zellen im einzelnen zu Spaltöffnungsinitialen werden, wie es z. B. bei dem von $\mathrm{S}$ a g r o $\mathrm{m} \mathrm{sk} \mathrm{y}^{2}$ untersuchten Gerbstoffmuster der Fall ist.

Schwieriger als bei den Spaltöffnungen ist es bei den Drüsen und Haaren, eine befriedigende Hypothese über die materiellen Voraussetzungen für $\mathrm{ihr}$
Zustandekommen aufzustellen. Die Tatsache, daß diese Emergenzen bei Grindelia allein in den stomatafreien Streifen über und unter den Leitbündeln auftreten, läßt darauf schließen, daß ihr Wuchsstoffbedarf und ihr $p_{\mathrm{H}}$-Optimum von denen der Stomata abweichen. Daß es nicht gelang, durch Wuchsstoffpaste die Epidermis über einem Palisadenparenchymfeld zur Bildung einer Drüse zu veranlassen, beweist nicht unbedingt das Gegenteil, und wenn der Blattgrund der inneren Involucralblätter von Grindelia squarrosa auf Ober- und Unterseite weder über Spaltöffnungen noch über Emergenzen verfügt (vgl. $\mathrm{Napp-Zinn}{ }^{3}$ ), so könnte man Aciditäts- und Wuchsstoffverhältnisse annehmen, die weder für die Entstehung von Emergenzen noch für die Spaltöffnungsbildung geeignet sind. Auch in dieser Beziehung sind noch weitere Untersuchungen erforderlich.

\title{
Papierchromatographischer Nachweis der Aminosäureausscheidung in vitro kultivierter Maiswurzeln
}

\author{
Von Otto KandleR \\ Aus dem Botanischen Institut der Universität München \\ (Z. Naturforschg. 6 b, 137-445 [1951]; eingegangen am 6. September 1951)
}

1. Maiswurzeln wurden unter sterilen Bedingungen in einer Mineralsalze und Glucose enthaltenden Nährlösung kultiviert und nach verschiedener Kulturdauer die Nährlösung papierchromatographisch auf Aminosäuren untersucht. Dabei konnten folgende Amide und Aminosäuren nachgewiesen werden: Glutamin, Asparagin, Alanin, Serin, Asparaginsäure, Valin, Glutaminsäure und Leucin.

2. Der Vergleich der ausgeschiedenen mit den innerhalb der Wurzel vorliegenden freien Aminosäuren ergab, daß keine Aminosäure bevorzugt aktiv ausgeschieden wird, sondern daß eine klare Proportionalität zwischen Ausscheidung und Konzentration im Inneren der Wurzeln besteht.

3. Bei Stickstoffmangel werden die anfänglich abgeschiedenen Aminosäuren wieder aufgenommen, während bei Stickstoffüberschuß die Menge der Aminosäuren in der Nährlösung im Laufe der Kultur weiter zunimmt.

4. Die Verteilung des Gesamtstickstoffes auf „Eiweißstickstoff“, „löslichen Stickstoff“ und „ausgeschiedenen Stickstoff“ wird für verschiedene Stickstoffernährung kurvenmäßig dargestellt.

5. Reiner Sauerstoff in der Gasphase fördert die Ausscheidung von Aminosäuren, Sauerstoffmangel unterdrückt sie.

6. In vitro kultivierte Spargelsprosse zeigten auch bei optimaler Stickstoffversorgung keinerlei Aminosäureausscheidung.

7. Unter gleichen Bedingungen kultivierte Maisembryonen ließen nur in einigen Fällen eine schwache Ausscheidung von Glutamin erkennen.

$\mathrm{N}$ ach den ersten Beobachtungen von $\mathrm{Liebig}$ und $\mathrm{Sachs}{ }^{1}$, daß die Wurzeln fähig sind, Kalksteine anzuätzen, wurde die Frage nach der chemischen Natur der Wurzelausscheidungen wiederholt aufgegriffen und bearbeitet. Neben $\mathrm{CO}_{2}$, das

1 J. v. Li e bi g, Liebigs Ann. Chem. 105, 109 [1858]; J. S a ch s, Bot. Z. 18, 117 [1860]. im wesentlichen für die ätzende Kraft der Wurzeln verantwortlich gemacht wird, dürften auch organische Säuren ${ }^{2}$, Zucker $^{3}$ und verschiedene Kationen aus-

2 F. C z a p e k, Jb. wiss. Bot. 29, 321 [1896]; J. S to$\mathrm{k} l$ a s a u. A. Ernes t, Jb. wiss. Bot. 46, 55 [1909].

3 Vgl. S. Kostyt s chew, Lehrbuch der Pflanzenphysiologie I, Berlin 1926. 